\title{
SHORT-TERM RED WINE CONSUMPTION PROMOTES DIFFERENTIAL EFFECTS ON PLASMA LEVELS OF HIGH-DENSITY LIPOPROTEIN CHOLESTEROL, SYMPATHETIC ACTIVITY, AND ENDOTHELIAL FUNCTION IN HYPERCHOLESTEROLEMIC, HYPERTENSIVE, AND HEALTHY SUBJECTS
}

\author{
Ana CMAndrade, Fernando HY Cesena,Fernanda M Consolim-Colombo, Silmara \\ R Coimbra, Alexandre M Benjó, Eduardo M Krieger, Protasio Lemos da Luz
}

doi: 10.1590/S1807-59322009000500011

\begin{abstract}
Andrade ACM, Cesena FHY, Consolim-Colombo FM, Coimbra SR, Benjó AM, Krieger EM, et al. Short-term red wine consumption promotes differential effects on plasma levels of high-density lipoprotein cholesterol, sympathetic activity, and endothelial function in hypercholesterolemic, hypertensive, and healthy subjects. Clinics. 2009;64(5):435-42.
\end{abstract}

OBJECTIVES: To compare the metabolic, hemodynamic, autonomic, and endothelial responses to short-term red wine consumption in subjects with hypercholesterolemia or arterial hypertension, and healthy controls.

METHODS: Subjects with hypercholesterolemia $(n=10)$ or arterial hypertension $(n=9)$, or healthy controls $(n=7)$ were given red wine $(250 \mathrm{~mL} / \mathrm{night})$ for 15 days. Analyses were performed before and after red wine intake.

RESULTS: Red wine significantly increased the plasma levels of HDL-cholesterol in the controls, but not in the other groups. The effects on hemodynamic measurements were mild, non-significantly more prominent in healthy subjects, and exhibited high interindividual variability. Across all participants, mean blood pressure decreased $7 \mathrm{mmHg}(\mathrm{p}<0.01)$ and systemic vascular resistance decreased $7 \%(\mathrm{p}=0.05)$. Heart rate and cardiac output did not significantly change in any group. Red wine enhanced muscle sympathetic fibular nerve activity in hypercholesterolemic and hypertensive patients, but not in controls. At baseline, brachial artery flow-mediated dilation was impaired in patients with hypercholesterolemia and arterial hypertension; red wine restored the dilation in the hypercholesterolemic group but not in the hypertensive group.

CONCLUSIONS: Red wine elicits different metabolic, autonomic, and endothelial responses among individuals with hypercholesterolemia or arterial hypertension and healthy controls. Our findings highlight the need to consider patient characteristics when evaluating the response to red wine.

KEYWORDS: Alcohol; Red Wine; Endothelial Function; Flow-mediated Dilation; Sympathetic Activity

\section{INTRODUCTION}

Several studies have documented a protective role of moderate wine consumption ( 15.5 to $31 \mathrm{~g}$ alcohol/day) in both vascular and nonvascular diseases. ${ }^{1-3}$ Different mechanisms may be responsible for these beneficial effects, including increases in HDL-cholesterol plasma levels, ${ }^{4}$

Heart Institute (InCor), Faculdade de Medicina da Universidade de São Paulo - São Paulo/SP, Brazil.

Email: ana.andrade@incor.usp.br

Tel.: 55113069.5237

Received for publication on February 27, 2009

Accepted for publication on March 04, 2009 decreased platelet aggregation, ${ }^{5}$ antioxidant effects, ${ }^{6}$ and restoration of endothelial function by flavonoids. ${ }^{7,8}$

On the other hand, high alcohol intake (> $31 \mathrm{~g}$ alcohol/ day) is associated with undesirable consequences, such as elevation of blood pressure $e^{9,10}$ and activation of the sympathetic system. ${ }^{11}$ Epidemiological studies consistently show a J-shaped relation between alcohol consumption and total mortality. ${ }^{3}$

We have previously shown that red wine and purple grape juice restore endothelial function in hypercholesterolemic individuals without any additional risk factors. ${ }^{8}$ It is not well known whether this beneficial effect is reproducible in people with other conditions that may be associated with 
endothelial dysfunction such as arterial hypertension. In fact, the influence of different risk factors on the various properties of red wine has not been fully investigated. Studies comparing the different effects of red wine in populations with different characteristics are lacking.

We have put forward the hypothesis that the effects of red wine on different aspects related to cardiovascular disease may differ according to individual characteristics, specifically whether the subject is hypertensive or hypercholesterolemic.

Hence, the purpose of this study was to compare the effects of 15 days of red wine consumption on the lipid profile, hemodynamic profile, sympathetic activity, and endothelial function of patients with hypercholesterolemia or arterial hypertension and in healthy controls.

\section{MATERIALS AND METHODS}

\section{Subjects and protocol}

Ten subjects with hypercholesterolemia (HCHOL group), nine with arterial hypertension (AH group), and seven with neither hypercholesterolemia nor arterial hypertension (healthy controls) were enrolled. Arterial hypertension was defined as blood pressure (BP) $>140 / 90 \mathrm{~mm} \mathrm{Hg}$, according to the Seventh Report of the Joint National Committee on Prevention, Detection, Evaluation, and Treatment of High Blood Pressure. ${ }^{12}$ Hypercholesterolemia was defined as a plasma LDL-cholesterol $>160 \mathrm{mg} / \mathrm{dL}$. None of the subjects had clinical evidence of any serious disease or condition that alters sympathetic activity such as diabetes mellitus; heart, liver, or renal failure; obesity; smoking; old age ( $>65$ years); or peripheral neuropathies. They were asked not to ingest products containing caffeine or polyphenols, such as fruit, coffee, tea, and alcoholic beverages other than the wine used in the study, during the 72 hours preceding the protocol examinations. They were encouraged not to change their daily dietary habits otherwise. They were not taking statins, and they were told to stop taking any antihypertensive drugs three weeks before the beginning of the protocol. Any possible harmful effects of medication withdrawal were monitored with periodic blood pressure (BP) measurements and active questioning of the subjects.

Blood collection (after a 12-hour overnight fast), analyses of endothelial function (in the morning) and sympathetic activity (at least 2 hours after lunch) were all conducted on the same day. Individuals were then provided with a supply of red wine (Varietal, Vinícola Aurora, Rio Grande do Sul, Brazil) and instructed to drink $250 \mathrm{~mL}$ each night, after a meal, for 15 consecutive days. After this period, evaluation of both endothelial function and sympathetic activity was repeated. Endothelial function was also reassessed 15 days after the discontinuation of red wine consumption (washout period) in 14 subjects.

Written informed consent was obtained from all participants. The ethics committee of the Heart Institute (InCor) and Hospital das Clínicas of the University of São Paulo Medical School approved the protocol.

\section{Blood analysis}

Plasma levels of total cholesterol, HDL-cholesterol, LDL-cholesterol, triglycerides, and glucose were determined by enzymatic methods (Roche Laboratories, Basel, Switzerland).

\section{Microneurography protocol}

Muscle sympathetic nerve activity (MSNA) was assessed by direct multiunit recordings of postganglionic sympathetic activity of the fibular nerve. The procedure involved selective insertion of unipolar tungsten microelectrodes into nerve fibers in the muscle, posterior to the fibular head, according to a previously described technique. ${ }^{13}$ Filtered and mean neurograms were recorded on millimetered paper. Muscle sympathetic bursts were identified by visual inspection by a single blinded investigator (F.C.C.) and were expressed as bursts per minute.

After placement of the electrode, the patient rested for 10 minutes to ensure that the BP, heart rate (HR), and MSNA were at basal levels. MSNA was then recorded for 10 minutes. After that, a cold pressor stimulus, consisting of immersion of the hand up to the wrist into ice water for 2 minutes, was initiated. After recording MSNA, this stimulus was followed by another 10-minute rest period to ensure that BP, HR, and MSNA returned to basal conditions. The subjects were then engaged in an isometric exercise, which involved pulling a dynamometer at $30 \%$ of the subject's maximum effort for 2 minutes, and MSNA was again determined.

\section{Hemodynamic variables}

During the microneurography protocol, HR, BP, and electrocardiography (DII lead) were continuously monitored. BP was measured noninvasively, beat-to-beat, using a photoplethysmographic finger device (Portapres, Finapres Medical Systems, The Netherlands). This method is based on a volume-clamp technique, and the mean BP is obtained as the integral of pressure over each beat divided by the corresponding beat interval. ${ }^{14,15}$ Measurements were transferred to a computer for calculation 
of several hemodynamic variables (including cardiac output and systemic vascular resistance) using commercial software (BeatScope 1.1, Finapres Medical Systems, The Netherlands).

\section{Brachial artery reactivity protocol}

Brachial artery ultrasound studies were performed to assess endothelial function by measuring flow-mediated dilation (FMD) induced by reactive hyperemia as previously described $^{8}$ and according to current guidelines. ${ }^{16}$ Studies were performed after the patient had rested for 10 minutes in a temperature-controlled room $\left(20^{\circ}\right.$ to $\left.25^{\circ} \mathrm{C}\right)$ using a 7.5-MHz linear array vascular ultrasound transducer and an APOGEE 800 plus ATL ultrasound system (ATL Ultrasound, Bothell, WA, USA).

The left brachial artery was imaged 2 to $15 \mathrm{~cm}$ above the elbow and scanned in longitudinal section with the focus zone set to the depth of the near wall. During image acquisition, anatomic landmarks such as fascial planes were noted to help record the same image of the artery throughout the study. Vessel diameter was measured using specific software developed at the Heart Institute (São Paulo, Brazil) that uses a semiautomatic approach to measure artery diameter based on an active contour technique supplemented by multiresolution analysis ${ }^{8}$. The blinded operator selected a region of interest ( 0.39 inch in longitudinal diameter) in 6 series of brachial artery images obtained from B-mode ultrasound. The distance between the near and far wall media-adventitia during diastole was obtained for all images, and the average was calculated.

Reactive hyperemia was induced by inflation of a pneumatic BP tourniquet, placed around the forearm, to a systolic BP of $250 \mathrm{~mm} \mathrm{Hg}$, followed by deflation after 5 minutes. FMD was calculated as the ratio of the brachial artery diameter after reactive hyperemia to the baseline diameter, expressed as percent variation.

Endothelium-independent vasodilation was assessed by administration of sublingual isosorbide dinitrate $(5 \mathrm{mg})$ and calculated in an analogous fashion. The average of two measurements was taken.

\section{Statistical analysis}

Continuous variables were reported as mean \pm standard deviation (SD), unless otherwise noted. Data were compared using the paired Student's $t$ test or analysis of variance (ANOVA) with repeated measurements, and post hoc analyses were performed to determine differences between two groups and/or two moments (for instance, after vs. before red wine intake).

Correlation analyses were also performed to assess interdependence between changes in different variables after red wine intake.

The level of significance was taken to be $p<0.05$ for all analyses.

\section{RESULTS}

\section{Clinical and laboratory data}

Table 1 summarizes the clinical and laboratory characteristics of the three groups before and after red wine consumption. Groups were comparable in terms of gender and age distributions. As expected, the HCHOL group exhibited higher plasma levels of total cholesterol and LDLcholesterol compared with the $\mathrm{AH}$ and control groups and higher plasma concentrations of triglycerides compared with controls. Both the AH and HCHOL groups exhibited higher glucose levels than the controls, and the hypertensive

Table 1 - Clinical and laboratory data of patients before and after red wine intake

\begin{tabular}{|c|c|c|c|c|c|c|}
\hline & \multicolumn{2}{|c|}{ Hypercholesterolemia $(\mathrm{n}=10)$} & \multicolumn{2}{|c|}{ Arterial hypertension $(\mathrm{n}=9)$} & \multicolumn{2}{|c|}{ Control $(n=7)$} \\
\hline & Before red wine & After red wine & Before red wine & After red wine & Before red wine & After red wine \\
\hline Sex (men/women) & \multicolumn{2}{|c|}{$7 / 3$} & \multicolumn{2}{|c|}{$7 / 2$} & \multicolumn{2}{|c|}{$5 / 2$} \\
\hline Age (years) & \multicolumn{2}{|c|}{$43.2 \pm 6.9$} & \multicolumn{2}{|c|}{$45.1 \pm 7.4$} & \multicolumn{2}{|c|}{$37.3 \pm 7.2$} \\
\hline BMI $\left(\mathrm{kg} / \mathrm{m}^{2}\right)$ & $25.6 \pm 2.9$ & $25.8 \pm 3.0$ & $27.9 \pm 1.9^{\mathrm{d}}$ & $28.1 \pm 1.9$ & $24.5 \pm 2.9$ & $24.4 \pm 2.7$ \\
\hline Total cholesterol (mg/dL) & $259 \pm 19^{a}$ & $242 \pm 37$ & $199 \pm 45$ & $192 \pm 51$ & $172 \pm 23$ & $194 \pm 38$ \\
\hline LDL-cholesterol (mg/dL) & $181 \pm 17^{\mathrm{a}}$ & $164 \pm 31$ & $131 \pm 38$ & $117 \pm 39$ & $106 \pm 15$ & $119 \pm 27$ \\
\hline HDL-cholesterol (mg/dL) & $53 \pm 9$ & $49 \pm 9$ & $47 \pm 10$ & $48 \pm 10$ & $52 \pm 12$ & $59 \pm 16^{\mathrm{e}}$ \\
\hline Triglycerides (mg/dL) & $126 \pm 46^{b}$ & $139 \pm 66$ & $100 \pm 36$ & $131 \pm 51^{\mathrm{e}}$ & $70 \pm 24$ & $81 \pm 30$ \\
\hline Glucose $(\mathrm{mg} / \mathrm{dL})$ & $97 \pm 9$ & $99 \pm 10$ & $97 \pm 8$ & $101 \pm 9$ & $88 \pm 7^{\mathrm{c}}$ & $91 \pm 5$ \\
\hline
\end{tabular}

BMI: body mass index; Data are reported as mean $\pm \mathrm{SD} ;{ }^{\mathrm{a}} \mathrm{p}<0.01 \mathrm{vs}$. AH or control before red wine; ${ }^{\mathrm{b}} \mathrm{p}=0.04 \mathrm{vs}$. control before red wine; ${ }^{\mathrm{c}} \mathrm{p} \leq 0.04 \mathrm{vs}$. $\mathrm{AH}$ or $\mathrm{HCHOL}$ before red wine; ${ }^{\mathrm{d}} \mathrm{p}=0.03$ vs. control before red wine; ${ }^{\mathrm{e}} \mathrm{p}=0.02 \mathrm{vs}$. before red wine 
individuals recorded a higher average body mass index than the controls.

When all patients were grouped together, red wine intake significantly increased plasma concentrations of triglycerides $(21 \%, \mathrm{p}<0.01)$ and glucose $(3 \%, \mathrm{p}=0.03)$. A significant elevation in HDL-cholesterol plasma levels after red wine intake was observed only in the healthy controls $(14 \%, \mathrm{p}=$ $0.02)$.

\section{Hemodynamic measurements and sympathetic activity}

\section{Resting condition}

Before red wine intake, systolic BP was significantly higher in the $\mathrm{AH}$ group compared with controls. Also, diastolic BP was nonsignificantly higher in the AH group than in the other groups, and, as expected, mean BP was significantly higher in the AH group than in the other groups (Table 2). This finding was not associated with significant differences in resting MSNA among the three groups (Table 3).

Although red wine markedly increased resting MSNA in the HCHOL and $\mathrm{AH}$ groups, the variation in healthy controls was minimal (Table 3). Indeed, after red wine intake, the peripheral sympathetic system was significantly more activated in the hypertensive and hypercholesterolemic individuals than in the healthy controls (Table 3 ).
In contrast with this sharp increase in sympathetic activity in the HCHOL and $\mathrm{AH}$ groups, the hemodynamic consequences of red wine were mild, non-significantly more prominent in healthy subjects (Table 2), and characterized by a high interindividual variability, especially in the HCHOL and $\mathrm{AH}$ groups.

Grouping all the patients together, red wine reduced systolic BP from $143 \pm 13 \mathrm{mmHg}$ to $138 \pm 13 \mathrm{mmHg}$ (p $=0.01$ ), diastolic BP from $84 \pm 9 \mathrm{mmHg}$ to $78 \pm 8 \mathrm{mmHg}$ ( $\mathrm{p}<0.01$ ), and mean BP from $108 \pm 11 \mathrm{mmHg}$ to $101 \pm 10$ $\mathrm{mmHg}(\mathrm{p}<0.01)$. This effect was likely driven by a fall in systemic vascular resistance, which decreased $7 \%$ across all patients ( $\mathrm{p}=0.05$ vs. before red wine), $1 \%$ in the HCHOL group, $8 \%$ in the $\mathrm{AH}$ group, and $17 \%$ in the controls ( $\mathrm{p}=\mathrm{ns}$ for comparison among groups). No significant change was found in the variation of the cardiac output or HR after red wine consumption in any group (Table 2).

No correlation was detected between the variation in resting MSNA (after vs. before red wine) and either the change in mean BP or systemic vascular resistance.

\section{Effects of cold stimulus}

As expected, cold stimulus increased MSNA (26\% before, $36 \%$ after red wine intake, $\mathrm{p} \leq 0.01 \mathrm{vs}$. resting condition, across all patients).

Before the red wine intake, cold stimulus caused systolic

Table 2 - Hemodynamic data at rest, before and after red wine intake

\begin{tabular}{|c|c|c|c|c|c|c|}
\hline & \multicolumn{2}{|c|}{ Hypercholesterolemia $(n=10)$} & \multicolumn{2}{|c|}{ Arterial hypertension $(n=9)$} & \multicolumn{2}{|c|}{ Control $(n=7)$} \\
\hline & Before red wine & After red wine & Before red wine & After red wine & Before red wine & After red wine \\
\hline Heart rate (bpm) & $68 \pm 9$ & $68 \pm 11$ & $76 \pm 14$ & $75 \pm 10$ & $69 \pm 13$ & $71 \pm 6$ \\
\hline Systolic BP (mmHg) & $141 \pm 15$ & $135 \pm 12$ & $153 \pm 8^{\mathrm{a}}$ & $149 \pm 8^{c}$ & $134 \pm 6$ & $129 \pm 8$ \\
\hline Diastolic BP (mmHg) & $80 \pm 11$ & $75 \pm 7$ & $89 \pm 5$ & $85 \pm 6^{c}$ & $83 \pm 7$ & $73 \pm 7^{e}$ \\
\hline Mean BP (mmHg) & $104 \pm 13$ & $97 \pm 8$ & $116 \pm 6^{b}$ & $111 \pm 6^{\mathrm{d}}$ & $103 \pm 9$ & $94 \pm 6^{\mathrm{e}}$ \\
\hline Cardiac output (L/min) & $6.32 \pm 1.25$ & $6.14 \pm 1.08$ & $6.19 \pm 0.98$ & $6.55 \pm 1.26$ & $5.70 \pm 1.37$ & $6.46 \pm 1.27$ \\
\hline $\begin{array}{l}\text { Systemic vascular resistance } \\
\left(\text { dyne.s.cm }{ }^{-5}\right)\end{array}$ & $1037 \pm 291$ & $981 \pm 170$ & $1208 \pm 283$ & $1072 \pm 283$ & $1158 \pm 386$ & $919 \pm 245$ \\
\hline
\end{tabular}

BP: blood pressure; Data are reported as mean $\pm \mathrm{SD} ;{ }^{\mathrm{a}} \mathrm{p}=0.01 \mathrm{vs}$. control before red wine; ${ }^{\mathrm{b}} \mathrm{p}<0.01 \mathrm{vs}$. HCHOL or control before red wine; ${ }^{\mathrm{c}} \mathrm{p} \leq 0.03$ vs. control after red wine; ${ }^{\mathrm{d}} \mathrm{p}<0.01 \mathrm{vs}$. HCHOL or control after red wine; ${ }^{\mathrm{e}} \mathrm{p} \leq 0.03 \mathrm{vs}$. before red wine

Table 3 - Muscle sympathetic nerve activity (bursts/min) before and after red wine intake

\begin{tabular}{|c|c|c|c|c|c|c|}
\hline & \multicolumn{2}{|c|}{ Hypercholesterolemia $(n=10)$} & \multicolumn{2}{|c|}{ Arterial hypertension $(n=9)$} & \multicolumn{2}{|c|}{ Control $(n=7)$} \\
\hline & Before red wine & After red wine & Before red wine & After red wine & Before red wine & After red wine \\
\hline Rest & $27.0 \pm 6.8$ & $49.9 \pm 5.5^{\mathrm{a}}$ & $30.0 \pm 8.0$ & $42.7 \pm 9.6$ & $26.6 \pm 7.1$ & $26.9 \pm 8.8^{b}$ \\
\hline Cold stimulus & $31.2 \pm 6.5$ & $62.9 \pm 9.2^{\mathrm{a}}$ & $36.9 \pm 11.1$ & $58.1 \pm 11.9^{a}$ & $33.9 \pm 5.0$ & $37.4 \pm 6.6^{\mathrm{c}}$ \\
\hline Isometric exercise & $34.4 \pm 9.1$ & $55.8 \pm 10.9^{a}$ & $36.1 \pm 15.9$ & $51.2 \pm 13.6^{\mathrm{a}}$ & $31.1 \pm 5.8$ & $37.4 \pm 7.4^{\mathrm{d}}$ \\
\hline
\end{tabular}

Data are reported as mean $\pm \mathrm{SD} ;{ }^{\mathrm{a}} \mathrm{p} \leq 0.02$ vs. before red wine; ${ }^{\mathrm{b}} \mathrm{p} \leq 0.02 \mathrm{vs}$. HCHOL or AH at rest, after red wine; ${ }^{\mathrm{c}} \mathrm{p}<0.01 \mathrm{vs}$. HCHOL or AH at cold test, after red wine; ${ }^{\mathrm{d}} \mathrm{p}<0.01$ vs. HCHOL at isometric exercise, after red wine 
and diastolic BP to increase $21 \pm 10 \%$ and $19 \pm 10 \%$, respectively ( $\mathrm{p} \leq 0.01$ vs. resting condition, considering all patients). After consuming red wine, the corresponding values were $23 \pm 10 \%$ and $21 \pm 9 \%$ (p $\leq 0.01$ vs. resting condition, across all patients). There was no significant difference among the three groups in terms of blood pressure variation with the cold stimulus either before or after red wine intake.

Although it did not attain statistical significance, the increase in systemic vascular resistance with the cold stimulus was less pronounced after vs. before red wine, especially in healthy subjects. In the HCHOL group, the increase was $20 \%$ before and $12 \%$ after red wine; in the $\mathrm{AH}$ group, the numbers were $46 \%$ and $40 \%$, respectively; in controls, $22 \%$ and $7 \%$, respectively.

\section{Effects of isometric exercise}

As expected, isometric exercise increased MSNA (31\% before, $26 \%$ after red wine intake, $\mathrm{p} \leq 0.02$ vs. resting condition, across all patients). The effects of isometric exercise on hemodynamic measurements were more modest than those reported for the cold test. Before red wine intake, grouping all the patients together, both systolic and diastolic BP increased $7 \%(\mathrm{p}<0.01)$, and systolic vascular resistance increased $6 \%(\mathrm{p}=0.07)$. The magnitude of these isometric exerciseinduced variations was unchanged by red wine. In addition, no significant difference was observed among the groups.

\section{Brachial artery reactivity}

Before red wine intake, FMD was nonsignificantly lower in the HCHOL and $\mathrm{AH}$ groups compared to the controls (Figure 1). Red wine restored FMD in the HCHOL group, but not in the AH group. Endothelium-independent vasodilation was nonsignificantly impaired in the $\mathrm{AH}$ group compared with the HCHOL group and controls, both before or after red wine consumption.

We also sought to evaluate the subset of 14 individuals who performed the brachial artery reactivity test after a washout period. In five HCHOL subjects, the FMD was $6.9 \pm 12.3 \%$ at the beginning of the study, increased to $14.7 \pm 2.8 \%$ after red wine, and fell to $-0.4 \pm 7.4 \%$ after red wine washout, indicating, on average, an absence of FMD following red wine withdrawal. In seven hypertensive patients, FMD was $4.7 \pm 1.5 \%, 5.2 \pm 5.7 \%$, and $3.6 \pm$ $2.2 \%$ before red wine, after red wine, and after washout, respectively. In two healthy subjects, the respective dilations were $13.1 \pm 1.7 \%, 15.8 \pm 1.9 \%$, and $14.8 \pm 6.1 \%$.

There was no correlation between changes in either MSNA or systemic vascular resistance (after vs. before red wine intake) and variation in FMD.
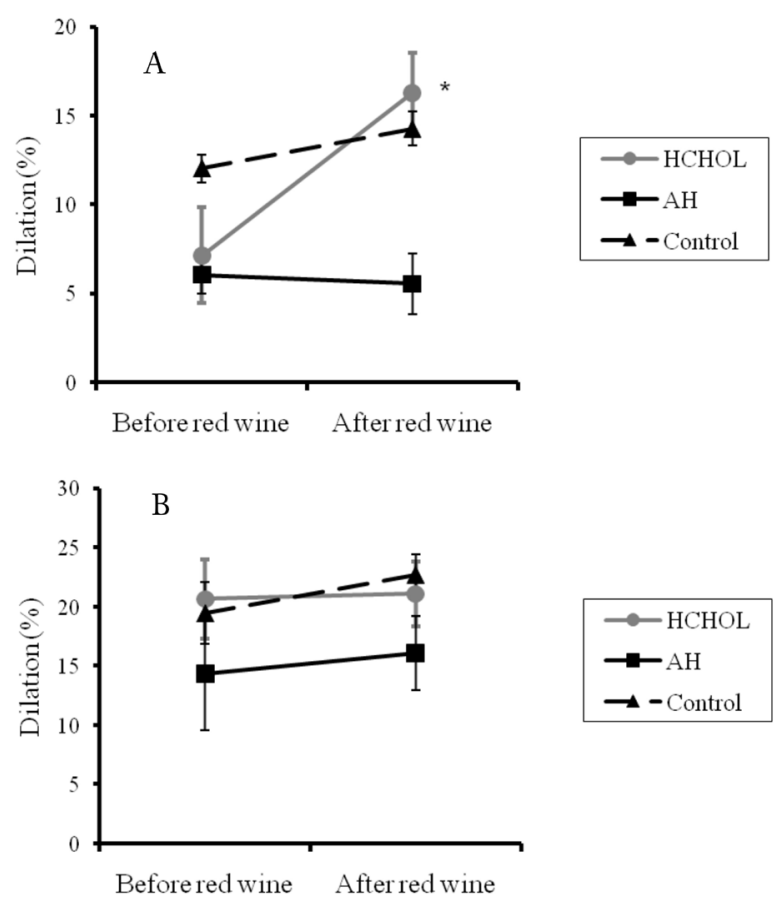

Figure 1 - Brachial artery flow-mediated (A) and nitroglycerin-mediated (B) dilation before and after red wine intake. Values are shown as means \pm standard errors. AH: arterial hypertension; HCHOL: hypercholesterolemia. $* \mathrm{p}=0.01$ before vs. after red wine in the HCHOL group

\section{DISCUSSION}

Numerous cross-sectional, observational, and controlled studies reveal a range of red wine effects on different aspects related to cardiovascular disease., ${ }^{3,17}$ Other studies show the relations of essential hypertension and coronary disease with genetic background, ${ }^{18}$ capillary density, ${ }^{19}$ and blood levels of cholesterol. ${ }^{20,21}$ Most of these studies have addressed one or a few outcomes, and not several at the same time, as in the current investigation. Moreover, most of the previous controlled trials have enrolled only healthy people ${ }^{17}$ and have not compared different populations simultaneously.

Our objectives were to compare the metabolic, hemodynamic, autonomic, and endothelial responses to red wine in subjects with hypercholesterolemia or arterial hypertension, or in healthy controls with neither of these risk factors. Several major findings are contributed by this study: (1) red wine increased plasma HDL-cholesterol levels only in the control group; (2) red wine slightly decreased mean $\mathrm{BP}$ in all groups, which was associated with a borderlinesignificant fall in systemic vascular resistance; (3) red wine caused a significant increase in sympathetic activity in the HCHOL and AH groups but not in controls; and (4) red wine restored FMD in the HCHOL group but not in the AH group. 


\section{Metabolic changes}

Several studies have documented the effects of alcoholic beverages on lipid metabolism. ${ }^{17}$ In particular, the HDLcholesterol-raising property of red wine seems to be primarily dependent on the alcoholic content, given that one head-to-head comparison shows that red wine, but not red grape extract, increases blood HDL-cholesterol concentrations. ${ }^{22}$

In a meta-analysis of studies evaluating different ethanolcontaining drinks in mostly healthy individuals, Rimm et al. ${ }^{17}$ reported that $30 \mathrm{~g}$ of ethanol per day increases the plasma concentration of HDL-cholesterol by $4 \mathrm{mg} / \mathrm{dL}$ and of triglycerides by $6 \mathrm{mg} / \mathrm{dL}$. Thus, the increase in triglyceride levels that we observed is consistent, although more than 3 -fold higher, than that reported by Rimm et al. ${ }^{17}$

The present study revealed that the increase in plasma HDL-cholesterol concentration was restricted to healthy subjects. In a previous investigation, we also observed no increase in HDL-cholesterol levels after 14 days of red wine ingestion in individuals with a high baseline plasma concentration of total cholesterol. ${ }^{8}$ One possible explanation for this finding is related to individuals being overweight or obese. In our cohort, healthy controls were thinner than the hypercholesterolemic or hypertensive patients. Interestingly, Hagiage et al. ${ }^{23}$ demonstrated that wine (30 g ethanol for 14 days) increases apolipoprotein A1 and decreases cholesteryl ester transfer protein activity in normoponderal but not in obese subjects, suggesting that obesity-associated abnormalities impair the effects of wine on HDL metabolism.

The very mild elevation in plasma glucose levels after red wine intake and the short-term nature of this study do not allow speculation as to the possible undesirable consequences of long-term alcohol intake on glucose metabolism. However, available data from the literature point in the opposite direction: population studies show that low-to-moderate regular alcohol intake decreases insulinemia and improves insulin sensitivity. ${ }^{24,25}$ In addition, alcohol consumption was recently inversely associated with the risk of type 2 diabetes in women in a case-control study. ${ }^{26}$

\section{Hemodynamic measurements}

Chronic heavy alcohol ingestion is consistently associated with hypertension. ${ }^{3}$ Although more controversial, moderate alcohol/red wine consumption is also believed to raise blood pressure, although an acute, transitory fall is reported to happen in the first hours after alcohol ingestion., ${ }^{9} 10,17$

Our study does not support the belief that moderate, short-term red wine consumption increases BP. Despite a high interindividual variability in our hemodynamic data that prevents definitive conclusions, red wine intake decreased the average BP in association with a quasi-significant fall in systemic vascular resistance. Interestingly, this decrease in $\mathrm{BP}$ was not observed in our previous investigation, using a very similar protocol. ${ }^{8}$ Differences in the type of wine used and the manner of measuring BP (standard pneumatic method in the forearm vs. plethysmographic finger device) may explain these contrasting results.

Importantly, the decrease in systemic vascular resistance cannot be attributed to changes in either sympathetic activity or endothelial function, suggesting a direct vasodilatory action of red wine. The less-pronounced elevation in systemic vascular resistance with the cold test after red wine consumption is also consistent with a vasodilatory property of red wine.

It is interesting to note that red wine increased sympathetic outflow only in the HCHOL and $\mathrm{AH}$ groups, whereas hemodynamic changes (decreases in BP and systemic vascular resistance) were nonsignificantly less expressive in these groups, as compared to controls. In addition, the attenuating effect of red wine on the increase in systemic vascular resistance with cold stimulus was more evident in healthy individuals. Although we found no correlation among variations (after vs. before red wine) in terms of MSNA, systemic vascular resistance, and FMD, these observations allow us to suggest that red wine decreases systemic vascular resistance and BP through a direct vasodilatory effect, but that activation of the sympathetic system (possibly by alcohol) blunts these hemodynamic effects.

\section{Sympathetic activity}

Previous studies ${ }^{27,28}$ have shown that acute alcohol or red wine ingestion increases sympathetic activity in healthy volunteers, in disagreement with the lack of increase in MSNA in our control group. It is possible that this discrepancy reflects the longer duration of and more moderate nature of alcohol intake in our study.

The reason why red wine increased MSNA in the $\mathrm{HCHOL}$ and $\mathrm{AH}$ groups but not in controls is not clear. Although speculative, it may be true that individuals with lipid disorders and those with hypertension share some abnormality that is responsible for the different effects of ethanol or red wine on autonomic activity, compared with normal individuals. A very reasonable candidate is the baroreflex dysfunction, which is known to be a feature of arterial hypertension and other conditions closely related to lipid abnormalities such as coronary artery disease, diabetes mellitus, obesity, and metabolic syndrome. ${ }^{29}$ Accordingly, 
baroreceptor impairment may be implicated in some ethanol effects. ${ }^{30}$ Therefore, the baroreflex dysfunction might be the link between red wine intake and increased MSNA in the HCHOL and AH groups, but not in healthy persons.

\section{Endothelial function}

The increase in FMD after red wine consumption in hypercholesterolemic subjects confirms our previous findings ${ }^{8}$ and supports the results of other studies that show acute benefits of wine or de-alcoholized red wine on the endothelium in patients with coronary artery disease..$^{31,32} \mathrm{In}$ the present study, in particular, the obvious fall in FMD after the washout period (this actually suggests a rebound effect) clearly supports a beneficial role of red wine components as they impact the endothelium in this population. This effect is most likely related to higher nitric oxide bioavailability. ${ }^{33,34}$

The lack of improvement in endothelial function in our hypertensive patients should be confirmed with further studies. Assuming endothelial dysfunction is an accepted marker of early atherosclerosis and has prognostic relevance, ${ }^{35}$ this finding has important clinical implications. We note, however, that endothelium-independent vasodilation was nonsignificantly lower in the AH group, which might have influenced the results of the reactive hyperemia.

Although our study failed to determine why the endothelial response to red wine was inconsistent between individuals with hypercholesterolemia or hypertension, we suggest that different mechanisms may account for endothelial dysfunction under such conditions. In support of this possibility, two studies from the same group have demonstrated that, in patients with essential hypertension, endothelium-dependent vasodilation in response to bradykinin is impaired. This contrasts with a preserved response to bradykinin in hypercholesterolemic subjects. ${ }^{36,37}$

\section{Limitations}

Our sample size may not have been sufficiently large to detect certain differences in the effects of red wine among the groups. We emphasize that this study evaluated the effects of short-term red wine intake, and our conclusions should not be extrapolated to longer-term ingestion scenarios. Endothelial function was analyzed using only one method, as was sympathetic activity. Mechanistic investigations, such as those involved in baroreflex dysfunction, were not performed. The lack of measurement of concentrations of resveratrol, catechin, or quercetin in plasma or urine does not allow us to reach firm conclusions regarding the effects of flavonoids in this study.

\section{CONCLUSIONS}

This study highlights the existence of significant variability in the effects of red wine on different patient groups. Elevation in HDL-cholesterol levels was noted only in healthy subjects, but not in those with arterial hypertension or high total cholesterol levels. Hemodynamic consequences of red wine intake were somewhat variable among subjects across all groups; on average, red wine decreased mean $\mathrm{BP}$, probably due to a direct vasodilatory action. Red wine activated the sympathetic system in individuals with hypercholesterolemia or arterial hypertension, but not in healthy controls. In terms of improving endothelial function, individuals with hypertension do not benefit from red wine as hypercholesterolemic subjects do.

Further investigations involving larger samples are required to confirm and understand the mechanisms involved in these findings and, consequently, to provide therapeutic implications. So far, the results reinforce the need for us to consider patient characteristics when evaluating the medical properties of red wine.

\section{ACKNOWLEDGEMENTS}

This study was supported by FAPESP - The State of São Paulo Research Foundation (number 03/09084-0) - São Paulo-SP, Brazil.

\section{REFERENCES}

1. da Luz PL, Coimbra SR. Wine, alcohol and atherosclerosis: clinical evidences and mechanisms. Braz J Med Biol Res. 2004;37:1275-95.

2. Klatsky AL, Friedman GD, Armstrong MA, Kipp H. Wine, liquor, beer, and mortality. Am J Epidemiol. 2003;158:585-95.

3. Opie LH, Lecour S. The red wine hypothesis: from concepts to protective signalling molecules. Eur Heart J. 2007;28:1683-93.

4. Nishiwaki M, Ishikawa T, Ito T, Shige H, Tomiyasu K, Nakajima K, et al. Effects of alcohol on lipoprotein lipase, hepatic lipase, cholesteryl ester transfer protein, and lecithin:cholesterol acyltransferase in high-density lipoprotein cholesterol elevation. Atherosclerosis. 1994;111:99-109.
5. Ruf JC. Alcohol, wine and platelet function. Biol Res. 2004;37:20915 .

6. Durak I, Avci A, Kaçmaz M, Büyükkoçak S, Cimen MY, Elgün S, et al. Comparison of antioxidant potentials of red wine, white wine, grape juice and alcohol. Curr Med Res Opin. 1999;15:316-20.

7. Stein JH, Keevil JG, Wiebe DA, Aeschlimann S, Folts JD. Purple grape juice improves endothelial function and reduces the susceptibility of LDL cholesterol to oxidation in patients with coronary artery disease. Circulation. 1999;100:1050-55. 
8. Coimbra SR, Lage SH, Brandizzi L, Yoshida V, da Luz PL. The action of red wine and purple grape juice on vascular reactivity is independent of plasma lipids in hypercholesterolemic patients. Braz J Med Biol Res. 2005:38:1339-47.

9. Zilkens RR, Burke V, Hodgson JM, Barden A, Beilin LJ, Puddey IB. Red wine and beer elevate blood pressure in normotensive men. Hypertension. 2005;45:874-9.

10. McFadden CB, Brensinger CM, Berlin JA, Townsend RR. Systematic review of the effect of daily alcohol intake on blood pressure. Am J Hypertens. 2005;18:276-86.

11. Grassi GM, Somers VK, Renk WS, Abboud FM, Mark AL. Effects of alcohol intake on blood pressure and sympathetic nerve activity in normotensive humans: a preliminary report. J Hypertens. Suppl 1989;7:S20-1.

12. Chobanian AV, Bakris GL, Black HR, Cushman WC, Green LA, Izzo JL $\mathrm{Jr}$, et al. Seventh report of the Joint National Committee on Prevention, Detection, Evaluation, and Treatment of High Blood Pressure. Hypertension. 2003;42:1206-52.

13. Delius W, Hagbarth KE, Hongell A, Wallin BG. General characteristics of sympathetic activity in human muscle nerves. Acta Physiol Scand. 1972;84:65-81.

14. Harms MP, Wesseling KH, Pott F, Jenstrup M, Van Goudoever J, Secher $\mathrm{NH}$ et al. Continuous stroke volume monitoring by modelling flow from non-invasive measurement of arterial pressure in humans under orthostatic stress. Clin Sci (Lond). 1999;97:291-301.

15. Imholz BP, Wieling W, van Montfrans GA, Wesseling KH. Fifteen years experience with finger arterial pressure monitoring: assessment of the technology. Cardiovasc Res. 1998;38:605-16.

16. Corretti MC, Anderson TJ, Benjamin EJ, Celermajer D, Charbonneau F, Creager MA, et al. International Brachial Artery Reactivity Task Force. Guidelines for the ultrasound assessment of endothelial-dependent flowmediated vasodilation of the brachial artery: a report of the International Brachial Artery Reactivity Task Force. J Am Coll Cardiol. 2002;39:25765 .

17. Rimm EB, Williams P, Fosher K, Criqui M, Stampfer MJ. Moderate alcohol intake and lower risk of coronary heart disease: meta-analysis of effects on lipids and haemostatic factors. BMJ. 1999;319:1523-8.

18. Lopes HF, Consolim-Colombo FM, Barreto-Filho JA, Riccio GM, Negrão CE, Krieger EM. Increased sympathetic activity in normotensive offspring of malignant hypertensive parents compared to offspring of normotensive parents. Braz J Med Biol Res. 2008;41:849-53.

19. Penna GL, Garbero R de F, Neves MF, Oigman W, Bottino DA, Bouskela E. Treatment of essential hypertension does not normalize capillary rarefaction. Clinics. 2008;63:613-8.

20. Crisostomo LM, Souza CA, Mendes CM, Coimbra SR, Favarato D, Luz PL. Vascular and metabolic response to statin in the mildly hypertensive hypercholesterolemic elderly. metabolic response to statin in the mildly hypertensive hypercholesterolemic elderly. Clinics. 2008;63:589-94.

21. Luz PL, Favarato D, Faria-Neto JR Jr, Lemos P, Chagas AC. High ratio of triglycerides to HDL-cholesterol predicts extensive coronary disease. Clinics. 2008:63:427-32.

22. Hansen AS, Marckmann P, Dragsted LO, Finné Nielsen IL, Nielsen SE, Grønbaek M. Effect of red wine and red grape extract on blood lipids, haemostatic factors, and other risk factors for cardiovascular disease. Eur J Clin Nutr. 2005;59:449-55.
23. Hagiage M, Marti C, Rigaud D, Senault C, Fumeron F, Apfelbaum $\mathrm{M}$, et al. Effect of a moderate alcohol intake on the lipoproteins of normotriglyceridemic obese subjects compared with normoponderal controls. Metabolism. 1992;41:856-61.

24. Kiechl S, Willeit J, Poewe W, Egger G, Oberhollenzer F, Muggeo $\mathrm{M}$, et al. Insulin sensitivity and regular alcohol consumption: large, prospective, cross sectional population study (Bruneck study). BMJ. 1996;313:1040-4

25. Freiberg MS, Cabral HJ, Heeren TC, Vasan RS, Curtis Ellison R; Third National Health and Nutrition Examination Survey. Alcohol consumption and the prevalence of the Metabolic Syndrome in the US: a cross-sectional analysis of data from the Third National Health and Nutrition Examination Survey. Diabetes Care. 2004;27:2954-9.

26. Beulens JW, Rimm EB, Hu FB, Hendriks HF, Mukamal KJ. Alcohol consumption, mediating biomarkers and risk of type 2 diabetes among middle-aged women. Diabetes Care. 2008;31:2050-5.

27. van de Borne P, Mark AL, Montano N, Mion D, Somers VK. Effects of alcohol on sympathetic activity, hemodynamics, and chemoreflex sensitivity. Hypertension. 1997;29:1278-83.

28. Spaak J, Merlocco AC, Soleas GJ, Tomlinson G, Morris BL, Picton P, et al. Dose-related effects of red wine and alcohol on hemodynamics, sympathetic nerve activity, and arterial diameter. Am J Physiol Heart Circ Physiol. 2008;294:H605-12.

29. Skrapari I, Tentolouris N, Katsilambros N. Baroreflex function: determinants in healthy subjects and disturbances in diabetes, obesity and metabolic syndrome. Curr Diabetes Ver. 2006;2:329-38.

30. Abdel-Rahman AA, Wooles WR. Ethanol-induced hypertension involves impairment of baroreceptors. Hypertension. 1987;10:67-73.

31. Whelan AP, Sutherland WH, McCormick MP, Yeoman DJ, de Jong SA, Williams MJ. Effects of white and red wine on endothelial function in subjects with coronary artery disease. Intern Med J. 2004;34:224-8.

32. Karatzi K, Papamichael C, Aznaouridis K, Karatzis E, Lekakis J, Matsouka $\mathrm{C}$ et al. Constituents of red wine other than alcohol improve endothelial function in patients with coronary artery disease. Coron Artery Dis. 2004; 15:485-90.

33. Andriambeloson E, Stoclet JC, Andriantsitohaina R. Mechanism of endothelial nitric oxide-dependent vasorelaxation induced by wine polyphenols in rat thoracic aorta. J Cardiovasc Pharmacol. 1999;33:24854

34. Puddey IB, Zilkens RR, Croft KD, Beilin LJ. Alcohol and endothelial function: a brief review. Clin Exp Pharmacol Physiol. 2001;28:10204.

35. Münzel T, Sinning C, Post F, Warnholtz A, Schulz E. Pathophysiology, diagnosis and prognostic implications of endothelial dysfunction. Ann Med. 2008;40:180-96.

36. Gilligan DM, Guetta V, Panza JA, García CE, Quyyumi AA, Cannon RO 3rd. Selective loss of microvascular endothelial function in human hypercholesterolemia. Circulation. 1994;90:35-41.

37. Panza JA, García CE, Kilcoyne CM, Quyyumi AA, Cannon RO 3rd. Impaired endothelium-dependent vasodilation in patients with essential hypertension. Evidence that nitric oxide abnormality is not localized to a single signal transduction pathway. Circulation. 1995;91:1732-8. 